\title{
Targeting diseased tissues by pHLIP insertion at low cell surface $\mathrm{pH}$
}

\author{
Oleg A. Andreev' , Donald M. Engelman ${ }^{2}$ and Yana K. Reshetnyak ${ }^{\text {* }}$ \\ ${ }^{1}$ Department of Physics, University of Rhode Island, Kingston, RI, USA \\ 2 Department of Molecular Biophysics and Biochemistry, Yale University, New Haven, CT, USA
}

\section{Edited by:}

Ebbe Boedtkjer, Aarhus University,

Denmark

Reviewed by:

Annarosa Arcangeli, University of

Florence, Italy

Luis M. S. Loura, University of

Coimbra, Portugal

*Correspondence:

Yana K. Reshetnyak, Department of Physics, University of Rhode

Island, 2 Lippitt Rd., Kingston,

RI 02881, USA

e-mail: reshetnyak@mail.uri.edu
The discovery of the $\mathrm{pH}$ Low Insertion Peptides (pHLIPs ${ }^{\circledR}$ ) provides an opportunity to develop imaging and drug delivery agents targeting extracellular acidity. Extracellular acidity is associated with many pathological states, such as those in cancer, ischemic stroke, neurotrauma, infection, lacerations, and others. The metabolism of cells in injured or diseased tissues often results in the acidification of the extracellular environment, so acidosis might be useful as a general marker for the imaging and treatment of diseased states if an effective targeting method can be developed. The molecular mechanism of a pHLIP peptide is based on pH-dependent membrane-associated folding. pHLIPs, being moderately hydrophobic peptides, have high affinities for cellular membranes at normal $\mathrm{pH}$, but fold and insert across membranes at low $\mathrm{pH}$, allowing them to sense $\mathrm{pH}$ at the surfaces of cells in diseased tissues, where it is the lowest. Here we discuss the main principles of pHLIP interactions with membrane lipid bilayers at neutral and low pHs, the possibility of tuning the folding and insertion $\mathrm{pH}$ by peptide sequence variation, and potential applications of pHLIPs for imaging, therapy and image-guided interventions.

Keywords: universal health test, imaging, nanotechnology, drug delivery
Many diseases such as cancer (solid tumors), ischemia, stroke, infection and others lead to the development of local hypoxia and acidosis. The extracellular acidosis results from enhanced use of glycolysis and production of carbonic and lactic acids, which are intensively pumped out cells to keep intracellular $\mathrm{pH}$ near neutral. Acids produced in this way accumulate in extracellular spaces since there is poor blood circulation in diseased tissues. As a consequence, a reversed membrane $\mathrm{pH}$ gradient is formed: the extracellular $\mathrm{pH}(\mathrm{pHe})$ in diseased tissue is lower than the intracellular $\mathrm{pH}$ ( $\mathrm{pHi}$ ) compared with normal tissues (Gerweck and Seetharaman, 1996; Raghunand et al., 1999). Extracellular acidity might serve as a general marker for detecting and targeting diseased tissue. However, such a strategy is challenging, since the bulk extracellular $\mathrm{pH}$ in diseased tissue is just $0.5-0.8 \mathrm{pH}$ units lower than the extracellular $\mathrm{pH}$ in healthy tissue (Hashim et al., 2011). From a biological standpoint the change is significant, and alters the functions and survival of cells. At the same time, from a chemical standpoint the change is small, so very precise tuning of chemical properties would be needed in a targeting agent. But, an important point that is often overlooked is that the $\mathrm{pH}$ is lowest at the surfaces of cells compared to the bulk extracellular $\mathrm{pH}$ (Chiche et al., 2010) and increases with distance from membrane, and becoming normal in the vicinity of blood vessels. So, the average $\mathrm{pH}$ in tissue is less informative than the $\mathrm{pH}$ at cellular surfaces, which might be the main target for the development of $\mathrm{pH}$-sensitive agents. We have been developing a novel class of $\mathrm{pH}$ sensitive delivery agents, $\mathrm{pHLIP}{ }^{\circledR} \mathrm{s}$ (pH Low Insertion Peptides), which are moderately hydrophobic peptides that can insert into membranes at mild acidic pHs, and which locate themselves at cell surfaces where the $\mathrm{pH}$ is lowest (Andreev et al., 2009, 2010a).

\section{MOLECULAR MECHANISM OF PHLIPS INTERACTION WITH MEMBRANE}

Peptides of the pHLIP family consist of flanking and transmembrane (TM) sequences (Figure 1A). The TM part is essential for the interaction with the membrane. The flanking-1 sequence is instrumental for peptide solubility. It usually contains polar and charged residues (Hunt et al., 1997; Reshetnyak et al., 2007; Barrera et al., 2011). The membrane-inserting flanking-2 sequence also can contribute to solubility, and affects the rates of peptide insertion and exit from the membrane (Karabadzhak et al., 2012). In general, peptides of the pHLIP family contain a mixture of natural and/or non-natural amino acids that are hydrophobic and protonatable at low $\mathrm{pH}$. The presence of hydrophobic residues ensures that the peptide maintains an affinity to membrane. The presence of protonatable residues is required (i) for guaranteeing solubility at neutral $\mathrm{pH}$, when they carry negative charges, and (ii) for the enhancement of hydrophobicity at low $\mathrm{pH}$, when the equilibrium is shifted toward protonation.

At neutral and high $\mathrm{pH}$, pHLIP is monomeric and largely unstructured. In the presence of a membrane or lipid bilayer, peptides in aqueous solution coexist with unstructured peptides adsorbed to the surface (Figure 1B). The fraction of the adsorbed peptides is controlled by the lipid:peptide ratio, which in turn affects diffusion of the peptide on membrane surface (Guo and Gai, 2010). Lowering the $\mathrm{pH}$ shifts the equilibrium toward folding, membrane insertion, and formation of a TM helix. A subsequent increase of $\mathrm{pH}$ promotes the reverse reaction: unfolding of the TM helix and its exit from the bilayer interior. Thus, peptide association with the membrane is distinguishable from 


\section{A AEQNPIY WARYADWLFTTPLLLLDLALLV DADEGT}

Flanking-1 Transmembrane $\quad$ Flanking-2

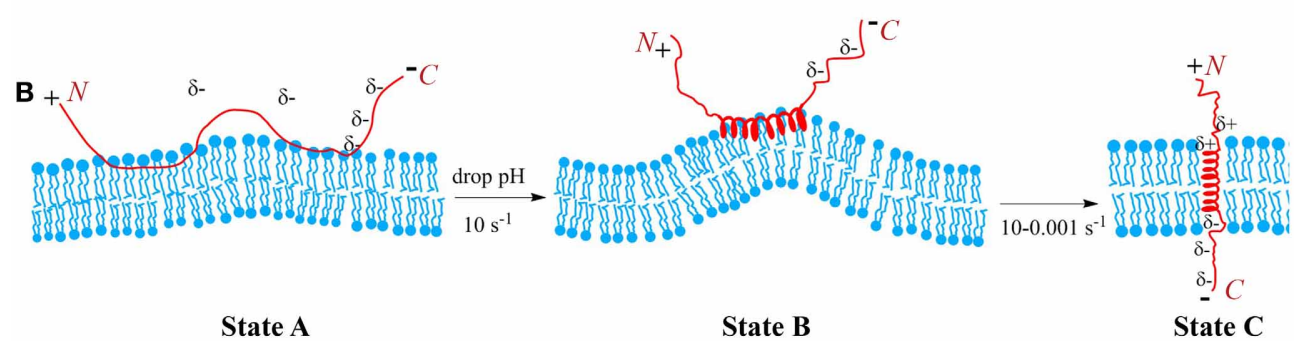

FIGURE 1 | Schematic presentation of pHLIP interaction with lipid bilayer of membrane. Sequence of the WT pHLIP (A). At high and neutral $\mathrm{pHs}$ pHLIP is associated with the lipid bilayer of membrane. Negative charges of Asp, Glu, and C-terminus prevent partition of the peptide into bilayer. After a drop of the $\mathrm{pH}$, some Asp/Glu residues are protonated, leading to an increase of overall peptide hydrophobicity that triggers deeper partitioning into the bilayer and the formation of an interfacial helix, which results in the distortion of the bilayer. Protonation of Asp/Glu at the inserting end (C-terminus) of the peptide leads to the formation of a transmembrane helix, which reduces the bilayer distortion (B). the process of peptide partitioning into the bilayer. The latter is accompanied by a coil-helix transition and triggered by a drop in $\mathrm{pH}$. Peptides consisting of L- or D-amino acids show $\mathrm{pH}$ dependent tumor cell targeting in vitro and in vivo confirming that the mechanism is TM helix formation (right or left handed, respectively), and that it does not depend on any specific recognition event such as binding to a receptor (Andreev et al., 2007; Macholl et al., 2012). The adsorption of pHLIPs to a model membrane surface is accompanied by an energy release of 5-6 $\mathrm{kcal} / \mathrm{mol}$, and the insertion process is accompanied by an additional energy release of about $1.8-2.5 \mathrm{kcal} / \mathrm{mol}$. Hence the bilayer affinity of the peptide is $30-50$ times higher at low $\mathrm{pH}$ than at high pH (Reshetnyak et al., 2008; Weerakkody et al., 2013). The pHLIP insertion results from the protonation of Asp/Glu residues in the TM part of the sequence and its (inserting) flanking-2 end. Carboxyl group protonation leads to an increase in hydrophobicity, which, in turn, triggers TM formation across the hydrophobic bilayer interior (Andreev et al., 2007; Musial-Siwek et al., 2010). Since the surface bound peptide is located at an intermediate zone between polar (aqueous) and non-polar (membrane) environments, the $\mathrm{pK}$ for the protonation of Asp and Glu residues is significantly shifted to higher $\mathrm{pH}$ values (Harris and Turner, 2002), and the apparent $\mathrm{pK}$ of pHLIP insertion can vary from 4.5 to 6.5 (Reshetnyak et al., 2007; Musial-Siwek et al., 2010; Barrera et al., 2011; Weerakkody et al., 2013).

pHLIP insertion is predominantly uni-directional. In most instances it is the C-terminus (flanking-2 end) that propagates across the bilayer and comes out in the cytoplasm (except of the reverse pHLIP sequence with an acetylated $\mathrm{N}$-terminus), while the N-terminus stays in the extracellular region (Reshetnyak et al., 2006; Thevenin et al., 2009). The propagation into the bilayer of the positively charged N-terminal at the flanking-1 end is energetically unfavorable compared to partition of the C-terminal at the flanking-2 end. The latter becomes electrically neutral after the protonation of $\mathrm{COO}^{-}$groups at low $\mathrm{pH}$ (Karabadzhak et al., 2012), while the positive charge is difficult to deprotonate and its passage is resisted by the membrane dipole potential. Peptide insertion into the membrane can be sub-divided into two distinct steps: (i) the formation of an interfacial helix and (ii) the movement of the helix across the bilayer to adopt a TM orientation. The timescale for the first process is about $0.1 \mathrm{~s}$, while for the second process it may vary from 0.1 up to $100 \mathrm{~s}$ (Andreev et al., 2010b; Karabadzhak et al., 2012), depending on several factors such as (i) the total number of protonatable residues in the sequence, (ii) their $\mathrm{pK}$ values, (iii) the presence of protonatable residues and/or polar cargo molecules at the peptide inserting end, and (iv) the compositional properties of the bilayer. The timescale for the peptide to exit from the bilayer varies from several milliseconds to seconds. It is also affected by the number of protonatable residues at the peptide inserting end, especially in the case of insertion into live cells, where the $\mathrm{pH}$ in the cytoplasm is 7.2-7.4. The Asp and Glu residues are moved across a bilayer while protonated, and in the cytoplasm they become de-protonated, i.e., negatively charged at pH7.2-7.4 and so serve as anchors for the peptide across a cell membrane, reducing significantly the rate of peptide exit from the bilayer. Thus, the number of protonatable groups on the peptide inserting end slows both insertion and exit rates.

The properties of the lipid bilayer itself play an important role in the process of peptide insertion. At neutral $\mathrm{pH}$, when a pHLIP is unstructured and associated with the outer leaflet of the lipid bilayer, it creates some tension and distortion of the bilayer (Figure 1B). However, due to the fact that the unstructured polypeptide cannot propagate very deep into the bilayer and due to the flexibility of the unstructured polypeptide at the surface of the membrane at high $\mathrm{pH}$, the distortion of the lipid bilayer is not sufficient to render state II, which is thermodynamically stable. However, when the peptide folds and adopts a more rigid, helical structure on the membrane surface (interfacial helical intermediate) the perturbation of the lipids is locally increased. The perturbation favors insertion, since a TM configuration is more compatible with the bilayer.

pHLIP, in contrast to cell-penetrating peptides, stays in the cellular membrane after insertion, translocating one end into the cytoplasm and leaving the other end in the extracellular 
space. Therefore, the peptide possesses dual delivery capabilities: it can tether cargo molecules to cell surfaces and/or it can inject and release cell-impermeable cargo molecules into cell cytoplasms (Andreev et al., 2009). In the first scenario, a cargo molecule, such as an imaging agent, is attached to the pHLIP's $\mathrm{N}$-terminus, remaining on the cell surface after pHLIP insertion. Transmembrane delivery by pHLIP is based on translocation of polar cargo molecules attached to the C-terminus, using a bond that is stable outside the cell, but cleaved in the cytoplasm. In addition, facilitator or quencher molecules can be attached to the C-terminal part of the peptide together with cargo and/or imaging agents (An et al., 2010; Wijesinghe et al., 2011). The chemical conjugation of various cargo molecules to pHLIPs is straightforward, since Lys and/or Cys residues, as well as other chemical functional moieties, can easily be included in the synthesis of the peptide.

\section{TARGETING OF ACIDIC DISEASED TISSUE}

Cancer cells acquire extensive genetic alterations as they divide in a tumor, including epigenetic regulation sites, point mutations, gene deletions, gene duplications, and chromosomal rearrangements. These changes are heterogeneously distributed within a single tumor (Gillies et al., 2012). The heterogeneity of expression of particular biomarkers at cell surfaces within a tumor and between tumors significantly reduces the effectiveness of agents that target specific biomarkers. On the other hand, low extracellular $\mathrm{pH}$, which is a hallmark of tumors and other pathological states, may provide a target independent of tumor heterogeneity, so agents like pHLIP are worth exploring.

The thermodynamics and kinetics of the pHLIP-membrane interaction predict preferential accumulation in acidic tissues. Indeed, pHLIP peptides conjugated with fluorescent dyes demonstrate excellent in vivo targeting of tumors of various origins (Andreev et al., 2007; Reshetnyak et al., 2011), ischemic myocardium (Sosunov et al., 2013), sites of inflammatory arthritis (Andreev et al., 2007), infection (Li et al., 2013) and ex vivo staining of cancerous tissue on biopsy samples (Loja et al., 2013). Clinical imaging modalities such as PET (positron emission tomography) and SPECT (single-photon emission computed tomography) also show tumor targeting by pHLIP-based probes (Vavere et al., 2009; Daumar et al., 2012; Macholl et al., 2012). pHLIPs consisting of D-amino acids have the same bilayer interactions as the L-amino acid versions, and show enhanced stability in vivo. Targeting of tumor acidity by pHLIP is correlated with MRS (magnetic resonance spectroscopy) measurements of low extracellular $\mathrm{pH}$ in tumors on live animals (Vavere et al., 2009). The extracellular acidity in tumors can be modulated by co-injection of glucose (which increases acidity through the Warburg effect) or feeding animals with bicarbonate water (which decreases acidity), resulting in enhanced or reduced pHLIP targeting of tumors, respectively (Vavere et al., 2009; Reshetnyak et al., 2011; Han et al., 2013). Analysis of pHLIP distribution in tumors over time shows that pHLIP can stay in tumors for several days, that tumor borders can be determined with high accuracy and that pHLIP is localized at tumor cell membranes (Segala et al., 2009; Reshetnyak et al., 2011). These properties suggest that fluorescent pHLIP-based agents could be used in image guided resections of tumors during surgery and in analysis of tissue samples.

Adaptation to hypoxia and acidosis may represent key events in the transition from in situ to invasive cancer (Gatenby and Gillies, 2007). Metastatic tumors, which have been shown to be more acidic, are labeled more effectively by pHLIP than non-metastatic ones (Reshetnyak et al., 2011). Further, ex vivo staining of biopsy samples correlates with stages of tumor development. The samples of normal tissue including those with chronic inflammation are not stained by pHLIP (Loja et al., 2013). Thus, there may be an opportunity to predict tumor invasiveness and distinguish aggressive tumor phenotypes. In addition to the targeting of primary tumors, the targeting of metastatic lesions by pHLIP has been demonstrated, and very small tumors $\left(\sim 1 \mathrm{~mm}^{3}\right)$ can be targeted (Reshetnyak et al., 2011). Recently we introduced a family of rationally designed $\mathrm{pHLIP}$ variants and demonstrated that tuning by variations of the peptide sequence and, as a result, the physical and chemical properties of peptide-membrane interactions, can modulate tumor targeting, blood clearance, and biodistribution (Weerakkody et al., 2013).

Since acidosis of different magnitudes is a general feature of a number of pathological states, and since the insertion $\mathrm{pK}$ of pHLIPs can be adjusted by sequence changes, a "universal health test" might be developed based on imaging by a spectrum of pHLIPs with different pKs. Various diseases might be identified at once and, each suspicious acidic spot could be investigated/diagnosed further.

\section{pHLIP AS A SINGLE-MOLECULE TRANSPORTER}

While targeting of diseased tissue is useful for imaging and diagnosis, an exciting use of pHLIP is for treatment using transport of an agent into a cell, where it can reach its cytoplasmic or nuclear target. Most inhibitors and all gene targeting agents are highly polar, and often, are charged molecules with extremely limited plasma membrane permeability. Several different approaches might be employed to move such molecules into a cell:

- modification of a drug molecule to reduce its polarity and enhance membrane-penetration;

- use of various nano-carriers;

- use of cell-penetrating peptides.

In conventional drug design and discovery the Lipinski rules of five (and other, related concepts) are widely used to guide molecular designs. The rules postulate that a successful drug should be hydrophobic and small in order to traverse membranes and reach cytoplasmic targets (e.g., the logarithm of the octanol-water partition coefficient $\log P_{o / w}$ is -0.4 to +5.6 and the MW is 160 to $480 \mathrm{gmol}^{-1}$ ) (Lipinski et al., 2001). There are several problems associated with this approach: (i) in some cases, it is difficult or even impossible to convert the polar/charged molecule into a hydrophobic one; (ii) modified drugs may lose their potency; (iii) drugs designed in this way will indiscriminately enter all cells they encounter, reducing effective concentrations in diseased areas and inducing side effects. The side effects can be especially devastating for cancer treatments, since the majority of the drugs are toxic molecules. 
Various nanosized drug-delivery vehicles including, but not limited to organic or inorganic nanoparticles, liposomes, micelles, viral particles, polymers, dendrimers, and others, have been designed for complexation or encapsulation of polar/charged therapeutic molecules. Nano-carriers usually are multifunctional, and targeting, imaging and therapeutic molecules can be combined in a single nano-carrier (Ferrari, 2005; Davis et al., 2008; Gindy and Prud'homme, 2009; Cheng et al., 2012; Han et al., 2013). Some targeting of vascularized tumors can result from the enhanced permeability of tumor vessels, resulting in preferential nanoparticle retention. Despite the advantages, there are significant limitations related to the use of nano-sized carriers, such as complexity and difficulty in manufacturing, reduced stability, potential toxicity of some nanomaterials, and reduced tumor-penetration ability. Significantly, all nanocarriers enter cells via one of the endocytotic routes, which are usually of low efficacy due to the entrapment of therapeutic molecule in the endosomal/lysosomal compartments.

Cell-penetrating, positively-charged peptides such as TAT [a peptide derived from the trans-activating transcriptional activator (TAT) protein], antennapedia, arginine-rich peptides and others, promote enhanced cellular uptake of molecules attached to them. The majority of cell-penetrating peptides enter cells via one of the endocytotic routes (Bechara and Sagan, 2013), although a number of reports indicate the possibility of direct diffusion across the membrane lipid bilayer. Commonly in these cases a hydrophobic motif is added to the peptide (Carrigan and Imperiali, 2005; Takayama et al., 2009), which, unfortunately, increases the probability of membrane destabilization and toxicity (Chan et al., 2006). Furthermore, cell-penetrating peptides are positively charged, which is also associated with the enhanced toxicity, limiting their use. Microbial peptides induce permeabilization of the plasma membranes and act cooperatively, which is non-advantageous for drug delivery systems. In each of these cases, there is no preferential tumor targeting, since the peptides enter both diseased and normal cells.

The folding and insertion into membrane of constitutive membrane proteins is facilitated by complex molecular machines in vivo, including the translocon that places most transmembrane helices across the bilayer (Van Den Berg et al., 2004; White and Von Heijne, 2004; Osborne et al., 2005). However, non-constitutive membrane proteins can insert transmembrane helices (TMs) into a lipid bilayer without assistance. Because the spontaneous insertion and folding of a TM peptide into a lipid bilayer approaches a free energy minimum, an insertion event is accompanied by the release of energy. The moderately hydrophobic TM forming peptides such pHLIPs might be considered as the basis for a novel class of delivery agents: single-molecular transporters. The membrane-associated folding of pHLIPs is accompanied by the release of about $2 \mathrm{kcal} / \mathrm{mol}$ of energy, which could be used as a biasing potential to favor the movement of polar, cell-impermeable cargo molecules across membrane into a cytoplasm. A significant feature of the process of peptide folding into a membrane is that it ensures a much higher cooperativity of the transition compared to the simple $\mathrm{pH}$-driven diffusion of weak bases across a membrane. Translocation is selective for low $\mathrm{pH}$, and various types of cargo molecules attached by disulfides to the inserting end of pHLIP (in most cases the C-terminus) can be transported into a cell and released in cytoplasm. We have demonstrated that pHLIP can translocate fluorescent dyes, cyclic peptides, polar toxins and peptide nucleic acids into cancer cells, and the properties of translocatable molecules can significantly violate Lipinski's rules, being larger and much more polar (Reshetnyak et al., 2006; Thevenin et al., 2009; An et al., 2010; Wijesinghe et al., 2011; Moshnikova et al., 2013). The translocation is fast (seconds to minutes), $\mathrm{pH}$ - and concentrationdependent and can be modulated by tuning of the hydrophobicity of the pHLIP inserting end. Compounds such as phalloidin and $\alpha$-amanitin have not been considered as anti-cancer therapeutic agents, since they cannot cross cellular membranes $\left(\log P_{o / w}\right.$ is about -1.5 and the $\mathrm{MW}$ is about $800-1000 \mathrm{gmol}^{-1}$ ). These toxins are selectively taken up by hepatocytes, leading to liver damage, since hepatocytes possess special transporting systems to translocate small cyclic molecules (Magdalan et al., 2010; Santi et al., 2012). Recently, it has been demonstrated that amanitin conjugated with an anti-human epithelial cell adhesion antibody induces inhibition of cell proliferation and suppression of pancreatic tumor growth (Moldenhauer et al., 2012). Conjugation of phallo- and amanita toxins to the pHLIP's inserting end via cleavable bond also leads to cell death in a low extracellular $\mathrm{pH}$ environment. The major mechanism of polar cargo delivery is direct translocation across the plasma membrane by pHLIP and cleavage of S-S bond in the reducing environment of the cytoplasm (other types of bonds might be used). Amanitin conjugated to pHLIP via non-cleavable cross-linkers or attached to the non-inserting end of the peptide was not able to induce cell death.

\section{pHLIP-MEDIATED DELIVERY OF NANOPARTICLES AND LIPOSOMES}

The pHLIP technology can also substantially improve the delivery of nanoparticles and liposomes to acidic diseased tissue (Davies et al., 2012; Sosunov et al., 2013; Wijesinghe et al., 2013; Yao et al., 2013a,b). The intratumoral and intravenous administration of gold nanoparticles conjugated with pHLIP both demonstrated 6-fold enhancement of tumor uptake compared to gold nanoparticles with no pHLIP (Yao et al., 2013a). Statistically significant reduction of gold accumulation was observed in acidic tumors and kidneys when $\mathrm{pH}$-nonsensitive K-pHLIP (where Asp residues are replaced by Lys) was used as a vehicle, suggesting an important role of $\mathrm{pH}$ in the $\mathrm{pHLIP}$-mediated targeting of gold nanoparticles. Gold nanoparticles delivered to a tumor might be used for local enhancement of radiation therapy.

We have also developed pH-sensitive, "fusogenic" pHLIPcoated liposomes (Wijesinghe et al., 2013; Yao et al., 2013b). The presence of pHLIP on the surfaces of liposomes enhances membrane fusion and lipid exchange in a $\mathrm{pH}$ dependent fashion, leading to an increase of cellular uptake and payload release, and consequent inhibition of cell proliferation by liposomes containing ceramide. Using two murine ischemia models we show that pHLIP-coated liposomes bind acidic ischemic but not normal regions of myocardium, while liposomes coated with PEG showed no preference in targeting of acidic ischemic tissue (Sosunov et al., 2013). 


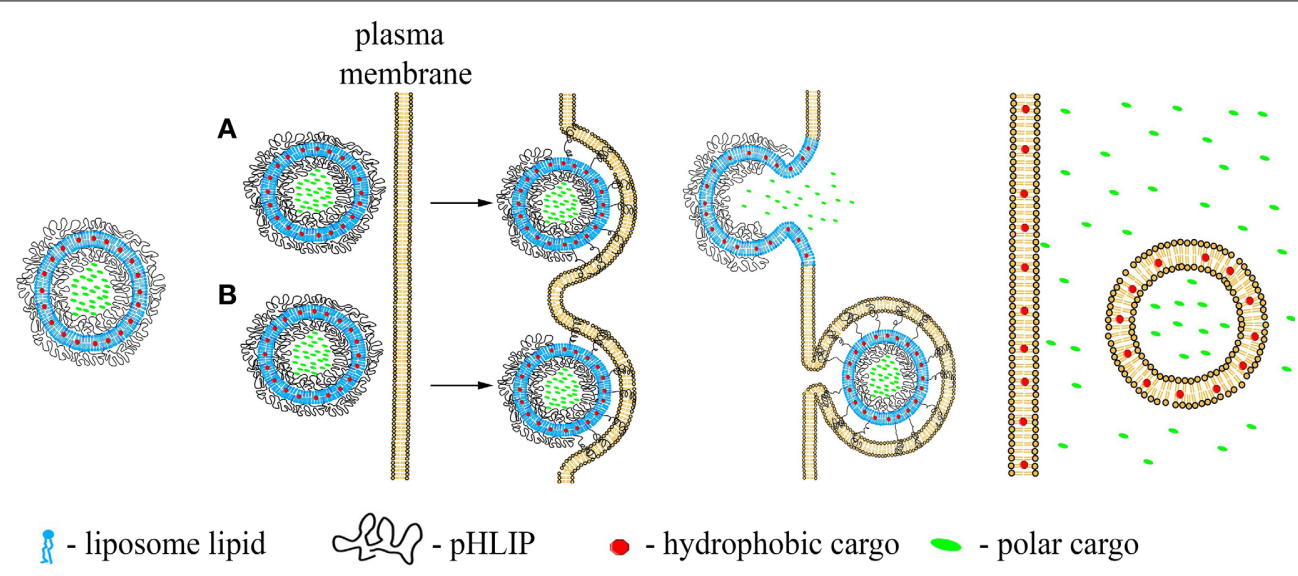

FIGURE 2 | Schematic presentation of pHLIP-coated liposome entry into a cell. pHLIP coated Liposomes with encapsulated polar cargo molecules or carrying hydrophobic cargoes in their bilayers can deliver payloads to cell membranes and cytoplasms. The acidity of diseased tissue results in pHLIP-mediated fusion of liposomes with plasma membranes at low extracellular $\mathrm{pH}(\mathbf{A})$ or fusion with endosomal membranes (B).
pHLIP-coated liposomes have been used for selective delivery of gramicidin nano-pores to cancer cells (Wijesinghe et al., 2013). Gramicidin channels inserted into the cancer cell membranes allow a flux of protons into the cytoplasm, and also disrupt the transmembrane balance of other monovalent cations, which induces cell apoptosis. Selective incorporation of functional gramicidin channels opens an opportunity for the delivery of other membrane peptides and proteins, which might find wide application in biotechnology and medicine.

In contrast to the pHLIP action as a single-molecule transporter when a single cargo molecule is attached to the single pHLIP peptide, multiple pHLIPs on the surfaces of liposomes or lipid-coated nanoparticles will interact with cellular membranes cooperatively, promoting membrane deformation, and tension. This binding will lead to lipid exchange, enhanced cellular uptake and/or direct fusion accompanied by the delivery of hydrophobic molecules to cellular membranes and polar cargo molecules to cytoplasm (Figures 2A,B). These processes are initiated in the acidic environment of diseased tissue, or, in the case of endocytotic uptake, will be even more effectively promoted by the low $\mathrm{pH}$ of endosomes/lysosomes, leading to the cytoplasmic release of cargo.

\section{CONCLUDING REMARKS}

Peptides of pHLIP family can be used to target cells diseased tissues as a result of extracellular acidity. Tuning the properties of pHLIP by sequence variation allows alteration of pharmacokinetics and targeting ability. pHLIPs conjugated with cell-impermeable cargoes are a novel class of delivery agents: single-molecule transporters for direct cytoplasmic delivery of polar cargo molecules into cells in acidic, diseased tissues. Multiple pHLIPs on the surfaces of nano-sized particles promote $\mathrm{pH}$-mediated distortion of cellular membranes, leading to the enhanced delivery of payloads to cytosols or cellular membranes.

\section{ACKNOWLEDGMENTS}

We are grateful to Mohan C. M. Arachchige for preparation of figures. The work was supported by the NIH grants CA133890 and GM073857 to Oleg A. Andreev, Donald M. Engelman and Yana K. Reshetnyak, and CA125280 Oleg A. Andreev and Yana K. Reshetnyak.

\section{REFERENCES}

An, M., Wijesinghe, D., Andreev, O. A., Reshetnyak, Y. K., and Engelman, D. M. (2010). pH-(low)-insertion-peptide (pHLIP) translocation of membrane impermeable phalloidin toxin inhibits cancer cell proliferation. Proc. Natl. Acad. Sci. U.S.A. 107, 20246-20250. doi: 10.1073/pnas.1014403107

Andreev, O. A., Dupuy, A. D., Segala, M., Sandugu, S., Serra, D. A., Chichester, C. O., et al. (2007). Mechanism and uses of a membrane peptide that targets tumors and other acidic tissues in vivo. Proc. Natl. Acad. Sci. U.S.A. 104, 7893-7898. doi: 10.1073/pnas.0702439104

Andreev, O. A., Engelman, D. M., and Reshetnyak, Y. K. (2009). Targeting acidic diseased tissue: new technology based on use of the $\mathrm{pH}$ (Low) Insertion Peptide (pHLIP). Chim. Oggi 27, 34-37.

Andreev, O. A., Engelman, D. M., and Reshetnyak, Y. K. (2010a). pH-sensitive membrane peptides (pHLIPs) as a novel class of delivery agents. Mol. Membr. Biol. 27, 341-352. doi: 10.3109/09687688.2010.509285

Andreev, O. A., Karabadzhak, A. G., Weerakkody, D., Andreev, G. O., Engelman, D. M., and Reshetnyak, Y. K. (2010b). pH (low) insertion peptide (pHLIP) inserts across a lipid bilayer as a helix and exits by a different path. Proc. Natl. Acad. Sci. U.S.A. 107, 4081-4086. doi: 10.1073/pnas.0914330107

Barrera, F. N., Weerakkody, D., Anderson, M., Andreev, O. A., Reshetnyak, Y. K., and Engelman, D. M. (2011). Roles of carboxyl groups in the transmembrane insertion of peptides. J. Mol. Biol. 413, 359-371. doi: 10.1016/j.jmb.2011.08.010

Bechara, C., and Sagan, S. (2013). Cell-penetrating peptides: 20 years later, where do we stand? FEBS Lett. 587, 1693-1702. doi: 10.1016/j.febslet.2013.04.031

Carrigan, C. N., and Imperiali, B. (2005). The engineering of membrane-permeable peptides. Anal. Biochem. 341, 290-298. doi: 10.1016/j.ab.2005.03.026

Chan, D. I., Prenner, E. J., and Vogel, H. J. (2006). Tryptophan- and arginine-rich antimicrobial peptides: structures and mechanisms of action. Biochim. Biophys. Acta 1758, 1184-1202. doi: 10.1016/j.bbamem.2006.04.006

Cheng, Z., Al Zaki, A., Hui, J. Z., Muzykantov, V. R., and Tsourkas, A. (2012). Multifunctional nanoparticles: cost versus benefit of adding targeting and imaging capabilities. Science 338, 903-910. doi: 10.1126/science.1226338

Chiche, J., Brahimi-Horn, M. C., and Pouyssegur, J. (2010). Tumour hypoxia induces a metabolic shift causing acidosis: a common feature in cancer. J. Cell. Mol. Med. 14, 771-794. doi: 10.1111/j.1582-4934.2009.00994.x 
Daumar, P., Wanger-Baumann, C. A., Pillarsetty, N., Fabrizio, L., Carlin, S. D., Andreev, O. A., et al. (2012). Efficient (18)F-labeling of large 37-amino-acid pHLIP peptide analogues and their biological evaluation. Bioconjug. Chem. 23, 1557-1566. doi: 10.1021/bc3000222

Davies, A., Lewis, D. J., Watson, S. P., Thomas, S. G., and Pikramenou, Z. (2012). pH-controlled delivery of luminescent europium coated nanoparticles into platelets. Proc. Natl. Acad. Sci. U.S.A. 109, 1862-1867. doi: 10.1073/pnas.1112132109

Davis, M. E., Chen, Z. G., and Shin, D. M. (2008). Nanoparticle therapeutics: an emerging treatment modality for cancer. Nat. Rev. Drug Discov. 7, 771-782. doi: $10.1038 / \operatorname{nrd} 2614$

Ferrari, M. (2005). Cancer nanotechnology: opportunities and challenges. Nat. Rev. Cancer 5, 161-171. doi: 10.1038/nrc1566

Gatenby, R. A., and Gillies, R. J. (2007). Glycolysis in cancer: a potential target for therapy. Int. J. Biochem. Cell Biol. 39, 1358-1366. doi: 10.1016/j.biocel.2007.03.021

Gerweck, L. E., and Seetharaman, K. (1996). Cellular pH gradient in tumor versus normal tissue: potential exploitation for the treatment of cancer. Cancer Res. 56, 1194-1198.

Gillies, R. J., Verduzco, D., and Gatenby, R. A. (2012). Evolutionary dynamics of carcinogenesis and why targeted therapy does not work. Nat. Rev. Cancer 12, 487-493. doi: 10.1038/nrc3298

Gindy, M. E., and Prud'homme, R. K. (2009). Multifunctional nanoparticles for imaging, delivery and targeting in cancer therapy. Expert Opin. Drug Deliv. 6, 865-878. doi: 10.1517/17425240902932908

Guo, L., and Gai, F. (2010). Heterogeneous diffusion of a membrane-bound pHLIP peptide. Biophys. J. 98, 2914-2922. doi: 10.1016/j.bpj.2010.03.050

Han, L., Ma, H., Guo, Y., Kuang, Y., He, X., and Jiang, C. (2013). pH-controlled delivery of nanoparticles into tumor cells. Adv. Healthc. Mater. 2, 1435-1439. doi: 10.1002/adhm.201300013

Harris, T. K., and Turner, G. J. (2002). Structural basis of perturbed pKa values of catalytic groups in enzyme active sites. IUBMB Life 53, 85-98. doi: $10.1080 / 15216540211468$

Hashim, A. I., Zhang, X., Wojtkowiak, J. W., Martinez, G. V., and Gillies, R. J. (2011). Imaging $\mathrm{pH}$ and metastasis. NMR Biomed. 24, 582-591. doi: $10.1002 / \mathrm{nbm} .1644$

Hunt, J. F., Earnest, T. N., Bousche, O., Kalghatgi, K., Reilly, K., Horvath, C., et al. (1997). A biophysical study of integral membrane protein folding. Biochemistry 36, 15156-15176. doi: 10.1021/bi970146j

Karabadzhak, A. G., Weerakkody, D., Wijesinghe, D., Thakur, M. S., Engelman, D. M., Andreev, O. A., et al. (2012). Modulation of the pHLIP transmembrane helix insertion pathway. Biophys. J. 102, 1846-1855. doi: 10.1016/j.bpj. 2012.03.021

Li, N., Yin, L., Thevenin, D., Yamada, Y., Limmon, G., Chen, J., et al. (2013). Peptide targeting and imaging of damaged lung tissue in influenza-infected mice. Future Microbiol. 8, 257-269. doi: 10.2217/fmb.12.134

Lipinski, C. A., Lombardo, F., Dominy, B. W., and Feeney, P. J. (2001). Experimental and computational approaches to estimate solubility and permeability in drug discovery and development settings. Adv. Drug Deliv. Rev. 46, 3-26. doi: 10.1016/S0169-409X(00)00129-0

Loja, M. N., Luo, Z., Greg Farwell, D., Luu, Q. C., Donald, P. J., Amott, D., et al. (2013). Optical molecular imaging detects changes in extracellular $\mathrm{pH}$ with the development of head and neck cancer. Int. J. Cancer 132, 1613-1623. doi: $10.1002 /$ ijc. 27837

Macholl, S., Morrison, M. S., Iveson, P., Arbo, B. E., Andreev, O. A., Reshetnyak, Y. K., et al. (2012). In vivo $\mathrm{pH}$ imaging with (99m)Tc-pHLIP. Mol. Imaging Biol. 14, 725-734. doi: 10.1007/s11307-012-0549-Z

Magdalan, J., Ostrowska, A., Piotrowska, A., Izykowska, I., Nowak, M., Gomulkiewicz, A., et al. (2010). alpha-Amanitin induced apoptosis in primary cultured dog hepatocytes. Folia Histochem. Cytobiol. 48, 58-62. doi: 10.2478/v10042-010-0010-6

Moldenhauer, G., Salnikov, A. V., Luttgau, S., Herr, I., Anderl, J., and Faulstich, H. (2012). Therapeutic potential of amanitin-conjugated anti-epithelial cell adhesion molecule monoclonal antibody against pancreatic carcinoma. J. Natl. Cancer Inst. 104, 622-634. doi: 10.1093/jnci/djs140

Moshnikova, A., Moshnikova, V., Andreev, O. A., and Reshetnyak, Y. K. (2013). Antiproliferative effect of pHLIP-amanitin. Biochemistry 52, 1171-1178. doi: $10.1021 /$ bi301647y
Musial-Siwek, M., Karabadzhak, A., Andreev, O. A., Reshetnyak, Y. K., and Engelman, D. M. (2010). Tuning the insertion properties of pHLIP. Biochim. Biophys. Acta 1798, 1041-1046. doi: 10.1016/j.bbamem.2009.08.023

Osborne, A. R., Rapoport, T. A., and Van Den Berg, B. (2005). Protein translocation by the Sec61/SecY channel. Annu. Rev. Cell Dev. Biol. 21, 529-550. doi: 10.1146/annurev.cellbio.21.012704.133214

Raghunand, N., Altbach, M. I., Van Sluis, R., Baggett, B., Taylor, C. W., Bhujwalla, Z. M., et al. (1999). Plasmalemmal pH-gradients in drug-sensitive and drugresistant MCF-7 human breast carcinoma xenografts measured by $31 \mathrm{P}$ magnetic resonance spectroscopy. Biochem. Pharmacol. 57, 309-312. doi: 10.1016/S00062952(98)00306-2

Reshetnyak, Y. K., Andreev, O. A., Lehnert, U., and Engelman, D. M. (2006). Translocation of molecules into cells by $\mathrm{pH}$-dependent insertion of a transmembrane helix. Proc. Natl. Acad. Sci. U.S.A. 103, 6460-6465. doi: 10.1073/pnas.0601463103

Reshetnyak, Y. K., Andreev, O. A., Segala, M., Markin, V. S., and Engelman, D. M. (2008). Energetics of peptide (pHLIP) binding to and folding across a lipid bilayer membrane. Proc. Natl. Acad. Sci. U.S.A. 105, 15340-15345. doi: 10.1073/pnas.0804746105

Reshetnyak, Y. K., Segala, M., Andreev, O. A., and Engelman, D. M. (2007). A monomeric membrane peptide that lives in three worlds: in solution, attached to, and inserted across lipid bilayers. Biophys. J. 93, 2363-2372. doi: 10.1529/biophysj.107.109967

Reshetnyak, Y. K., Yao, L., Zheng, S., Kuznetsov, S., Engelman, D. M., and Andreev, O. A. (2011). Measuring tumor aggressiveness and targeting metastatic lesions with fluorescent pHLIP. Mol. Imaging Biol. 13, 1146-1156. doi: 10.1007/s11307$010-0457-z$

Santi, L., Maggioli, C., Mastroroberto, M., Tufoni, M., Napoli, L., and Caraceni, P. (2012). Acute liver failure caused by amanita phalloides poisoning. Int. J. Hepatol. 2012, 487480. doi: 10.1155/2012/487480

Segala, J., Engelman, D. M., Reshetnyak, Y. K., and Andreev, O. A. (2009). Accurate analysis of tumor margins using a fluorescent ph low insertion peptide (pHLIP). Int. J. Mol. Sci. 10, 3478-3487. doi: 10.3390/ijms10083478

Sosunov, E. A., Anyukhovsky, E. P., Sosunov, A. A., Moshnikova, A., Wijesinghe, D., Engelman, D. M., et al. (2013). pH (low) insertion peptide (pHLIP) targets ischemic myocardium. Proc. Natl. Acad. Sci. U.S.A. 110, 82-86. doi: $10.1073 /$ pnas. 1220038110

Takayama, K., Nakase, I., Michiue, H., Takeuchi, T., Tomizawa, K., Matsui, H. et al. (2009). Enhanced intracellular delivery using arginine-rich peptides by the addition of penetration accelerating sequences (Pas). J. Control. Release 138, 128-133. doi: 10.1016/j.jconrel.2009.05.019

Thevenin, D., An, M., and Engelman, D. M. (2009). pHLIP-mediated translocation of membrane-impermeable molecules into cells. Chem. Biol. 16, 754-762. doi: 10.1016/j.chembiol.2009.06.006

Van Den Berg, B., Clemons, W. M. Jr., Collinson, I., Modis, Y., Hartmann, E., Harrison, S. C., et al. (2004). X-ray structure of a protein-conducting channel. Nature 427, 36-44. doi: 10.1038/nature02218

Vavere, A. L., Biddlecombe, G. B., Spees, W. M., Garbow, J. R., Wijesinghe, D., Andreev, O. A., et al. (2009). A novel technology for the imaging of acidic prostate tumors by positron emission tomography. Cancer Res. 69, 4510-4516. doi: 10.1158/0008-5472.CAN-08-3781

Weerakkody, D., Moshnikova, A., Thakur, M. S., Moshnikova, V., Daniels, J., Engelman, D. M., et al. (2013). Family of pH (low) insertion peptides for tumor targeting. Proc. Natl. Acad. Sci. U.S.A. 110, 5834-5839. doi: 10.1073/pnas.1303708110

White, S. H., and Von Heijne, G. (2004). The machinery of membrane protein assembly. Curr. Opin. Struct. Biol. 14, 397-404. doi: 10.1016/j.sbi. 2004.07.003

Wijesinghe, D., Arachchige, M. C., Lu, A., Reshetnyak, Y. K., and Andreev, O. A. (2013). pH dependent transfer of nano-pores into membrane of cancer cells to induce apoptosis. Sci. Rep. 3:3560. doi: 10.1038/srep03560

Wijesinghe, D., Engelman, D. M., Andreev, O. A., and Reshetnyak, Y. K. (2011). Tuning a polar molecule for selective cytoplasmic delivery by a pH (Low) insertion peptide. Biochemistry 50, 10215-10222. doi: 10.1021/ bi2009773

Yao, L., Daniels, J., Moshnikova, A., Kuznetsov, S., Ahmed, A., Engelman, D. M., et al. (2013a). pHLIP peptide targets nanogold particles to tumors. Proc. Natl. Acad. Sci. U.S.A. 110, 465-470. doi: 10.1073/pnas.1219665110 
Yao, L., Daniels, J., Wijesinghe, D., Andreev, O. A., and Reshetnyak, Y. K. (2013b). pHLIP(R)-mediated delivery of PEGylated liposomes to cancer cells. J. Control. Release 167, 228-237. doi: 10.1016/j.jconrel.2013. 01.037

Conflict of Interest Statement: The authors declare that the research was conducted in the absence of any commercial or financial relationships that could be construed as a potential conflict of interest.

Received: 29 October 2013; paper pending published: 17 December 2013; accepted: 25 February 2014; published online: 13 March 2014.
Citation: Andreev OA, Engelman DM and Reshetnyak YK (2014) Targeting diseased tissues by pHLIP insertion at low cell surface pH. Front. Physiol. 5:97. doi: 10.3389/ fphys.2014.00097

This article was submitted to Membrane Physiology and Membrane Biophysics, a section of the journal Frontiers in Physiology.

Copyright (c) 2014 Andreev, Engelman and Reshetnyak. This is an open-access article distributed under the terms of the Creative Commons Attribution License (CC BY). The use, distribution or reproduction in other forums is permitted, provided the original author(s) or licensor are credited and that the original publication in this journal is cited, in accordance with accepted academic practice. No use, distribution or reproduction is permitted which does not comply with these terms. 